\title{
The Versatility of Pedicled Anterolateral Thigh Flap: A Tertiary Referral Center Experience from India
}

\author{
Ravikiran Naalla, MCh; Shashank Chauhan, MCh; Sharmistha Bhattacharyya, MCh; Maneesh Singhal, MCh, \\ FRCS (Glasg)*
}

Department of Plastic Reconstructive \& Burns Surgery, All India Institute of Medical Sciences, New Delhi, India

\begin{abstract}
Background: Free anterolateral thigh flap (ALT) is considered as one of the most popular reconstructive options for soft tissue defects of multiple body regions. Though pedicled anterolateral thigh flap is useful to resurface soft tissue defects of multiple anatomical locations within its reach, its versatility is less reported.

Methods: Retrospective review was performed on 9 patients who underwent pedicled anterolateral thigh flap for coverage of soft tissue defects following trauma $(n=6)$, cancer resection $(n=3)$, and debridement of soft tissue infection $(n=1)$ during January 2016 to April 2017 . The anatomy \& designing of flap, pivot points, rotation arcs, and the outcomes were recorded.

Results: Pedicled anterolateral thigh flap was used to cover the soft tissue defects over the knee $(n=3)$, gluteal \& trochanteric region ( $n=3)$, abdomen $(n=2)$, and abdomen \& groin $(n=1)$. The flap was based on proximal and distal blood flow, depending upon the region. The rotation arc ranged from 100 to 180 degrees. All flaps survived, except one. Two patients had partial flap necrosis and one patient had hematoma at the flap site, which required drainage.

Conclusion: The pedicled anterolateral thigh flap is a versatile option for coverage of soft tissue defects within its reach.
\end{abstract}

\section{Introduction}

Anterolateral thigh (ALT) flap was first described by Song et al. [1]. Since then it has gained popularity and currently it is the work horse flap for soft tissue reconstruction of different anatomical regions. The versatility is due to its long vascular pedicle, ability to harvest different tissues in composite nature, large size of skin paddle, and less donor site morbidity. It can be used as sensate flap and flow through fashion, depending on the clinical situation. Feasibility of two team approaches [2] has decreased the operative time significantly reducing the complications associated with the longer general anaesthesia. The same characteristics also make ALT flap versatile in pedicled fashion [3].

The blood supply to the skin paddle of ALT flap is by septocutaneous or musculocutaneous perforators, which arise from the descending branch of lateral circumflex femoral artery (LCFA). The descending branch passes through the vastus lateralis muscle towards the knee and is anastomosed to lateral superior genicular artery or the profunda femoris artery perforators. Thus, ALT flap can be harvested based on either its proximal circulation or the distal circulation. This property, along with the long vascular pedicle, can be utilised to cover the various soft tissue defects involving the abdomen, groin, perineum, and trochanteric regions proximally (proximally based circulation), and soft tissue defects around the knee distally (distally based circulation) [3]. Though pedicled

*Correspondence: Maneesh Singhal, MCh, FRCS (Glasg)

Department of Plastic Reconstructive \& Burns surgery, All India Institute of Medical Sciences, New Delhi, India.

E-mail: drmaneesh@gmail.com

Received: Apr. 30, 2017; Accepted: Sep. 10, 2017; Published: Oct. 13, 2017

International Microsurgery Journal. 2017;1(2):5

DOI: 10.24983/scitemed.imj.2017.00040

Copyright ( 2017 The Author(s). This is an open-access article distributed under the terms of the Creative Commons Attribution 4.0 International License (CC-BY). anterolateral thigh flap is useful to resurface soft tissue defects of multiple anatomical locations within its reach, its versatility is less reported. In this article, we have shared our experience with the pedicled ALT flap for resurfacing complicated soft tissue defects within its reach. We have also stressed on the anatomy \& designing of flap, pivot points, rotation arcs, and the outcomes.

\section{Patients \& Methods}

Retrospective analysis of 9 patients, who underwent pedicled ALT during January 2016 to April 2017 for various soft tissue defects, was done. The medical records and photographic records were accessed. The details of patients including age, sex, nature of disease, defect location, structures covered/ reconstructed, location of the tunnel through which pedicle is routed, complications, and outcome were tabulated (Table 1).

\section{Technical Considerations}

The flap dissection was started only once the defect was made. The skin perforators of the anterolateral thigh flap were mapped with doppler pencil probe centered over the midpoint of a line connecting anterosuperior iliac spine and the superolateral border of the patella. The template of the soft tissue defect was measured and the flap was designed so that it is centered over the marked perforators, preferably. When the ALT flap was based proximally, the skin paddle had to be designed such that the flap reached the defect without stretching the vascular pedicle. Descending branch of LCFA or LCFA itself is usually the pivot point, depending upon the extent of dissection of the vascular pedicle. The distance between the skin perforator and the pivot point of the flap should closely match the distance between the pivot point and the central portion of the soft tissue defect.

Adrenaline mixed normal saline solution $(1: 2,00,000)$ was infiltrated in the flap margins. Dissection was started medially identifying deep fascia of thigh, followed by extension of the incision proximally and distally. All flaps were harvested in the sub-fascial plane. Dissection was proceeded laterally 


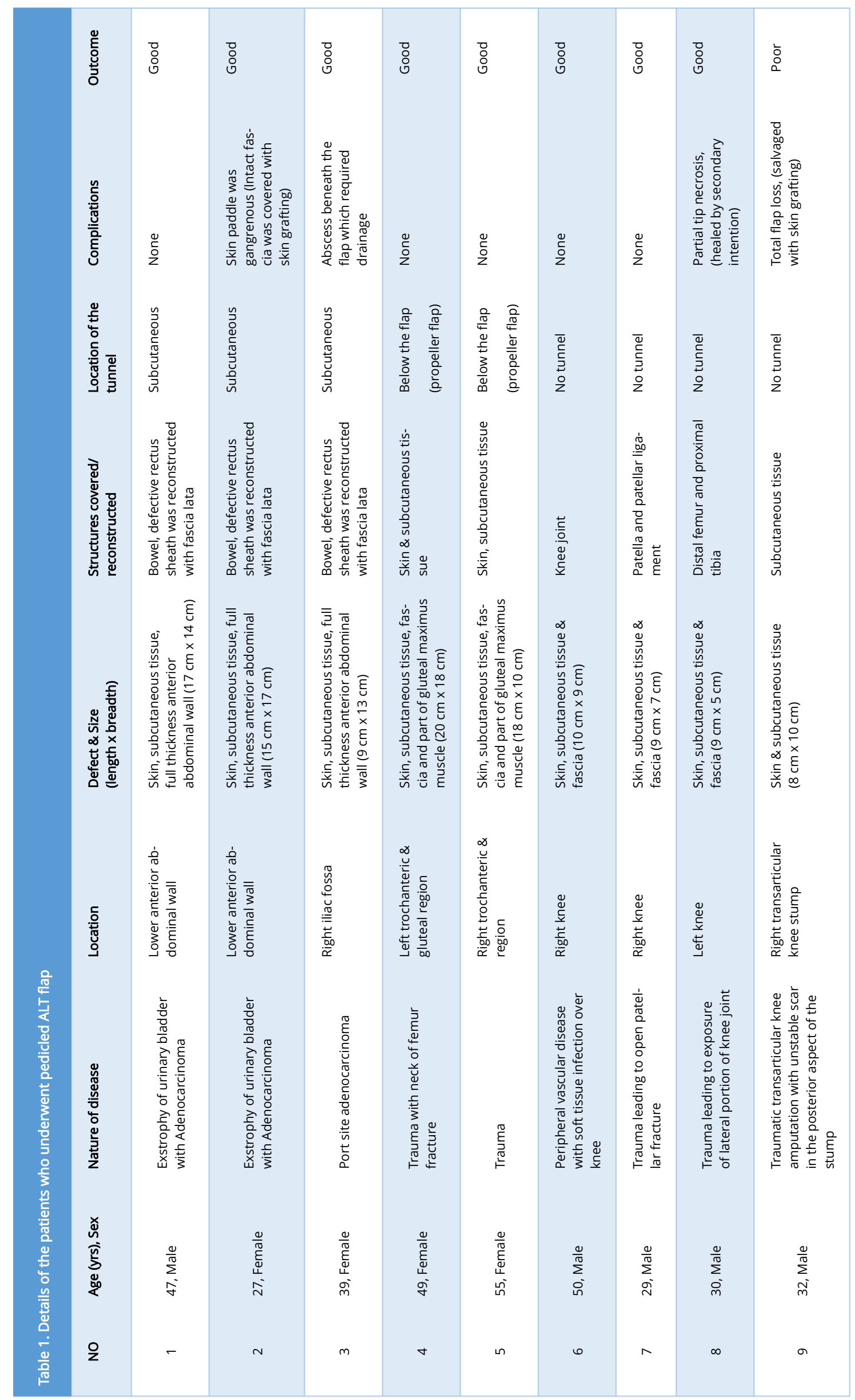




\section{Original}
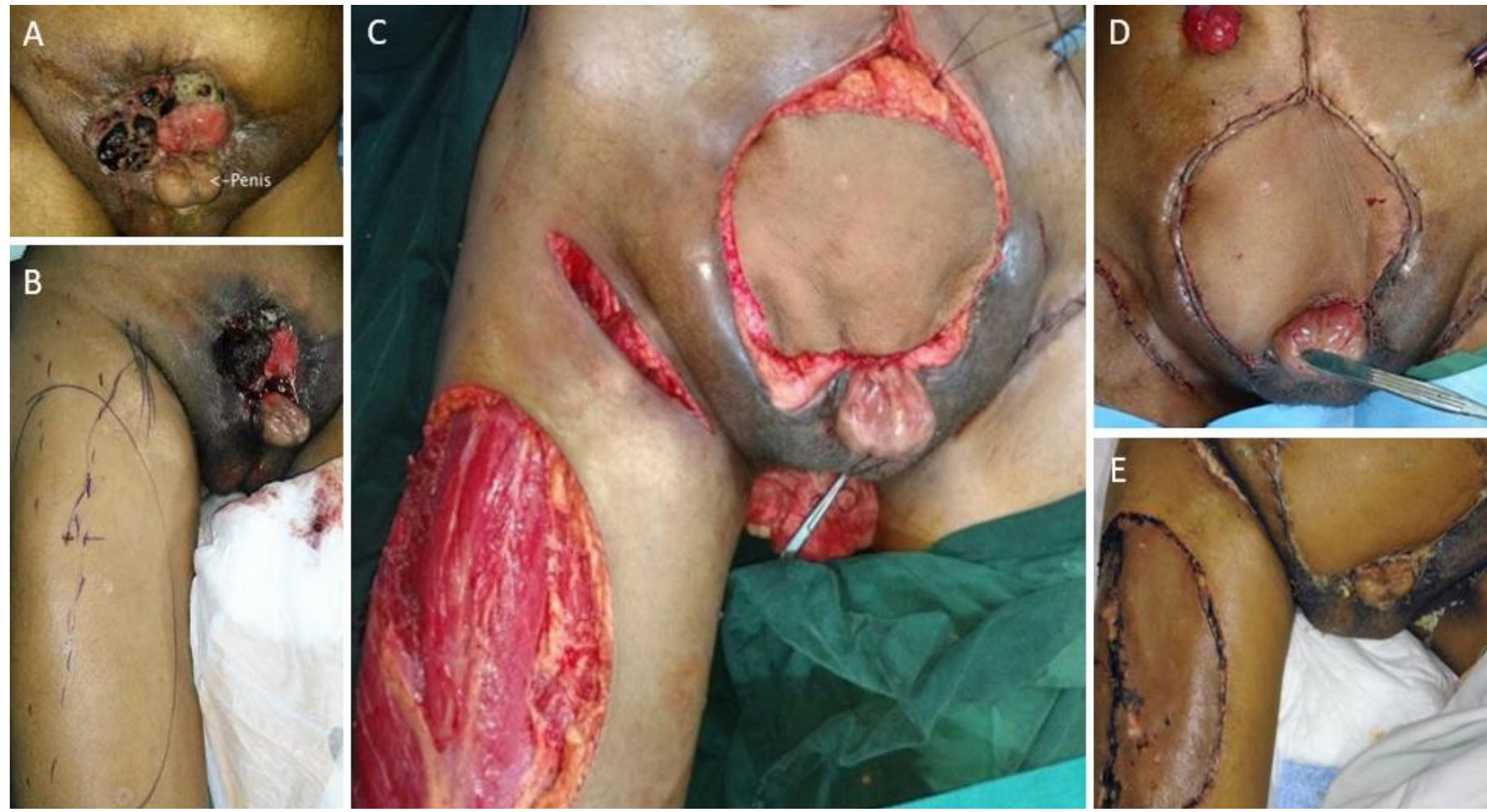

Figure 1. Image showing (A) exstrophy-epispadiasis complex \& ulcero-proliferative growth (adenocarcinoma of the urinary bladder) over the suprapubic region. (B) Preoperative marking of right anterolateral thigh flap. (C, D) Composite abdominal wall defect covered with ALT flap; the fascial lata was used to repair the fascial defect. Bilateral inguinal dissection wounds can be seen \& flap inset. (E) Well settled flap and donor site.

to identify the perforators entering the flap piercing the fascia. The dominant perforator was hooked around by a vessel sling. The septum between the rectus femoris and vastus lateralis was opened below upwards to identify the descending branch of the lateral circumflex femoral artery. The perforator to the flap was dissected intramuscularly by unroofing the vastus lateralis, unless a musculocutaneous flap was planned. The descending branch of LCFA was dissected till the origin. The transverse and the ascending branches could be divided to gain more length of the pedicle. Once the distance between the skin perforator and the pivot point matched the distance between the pivot point and the central portion of the defect to be reconstructed, the lateral margin of the flap was incised and dissected medially. The vessel loops hooked around the perforator helped in avoiding inadvertent injury to the perforator during lateral dissection. Flap perfusion was checked by assessing the subdermal blood flow. Vastus lateralis muscle, extended dimensions of vascularized fascial lata, and chimeric anteromedial thigh flaps can be harvested based on the requirements of the soft tissue defect to be reconstructed. Flap can be transposed into the defect to check the adequacy of the pedicle length. In case of deficient pedicle length of the flap, its reach can be increased when the pedicle is transposed below the rectus femoris and sartorius, especially when the flap has to reach the central portion of the abdominal wall. We prefer to tunnel the flap when we need to cover the abdominal and groin wounds, if the bridging tissue is adequate. We prefer to propel the flap, if the wound is close the flap and when the bridging tissue is insufficient to provide a tunnel. For coverage of knee wound, we prefer to divide the bridging skin to pass the pedicle to prevent mildest compression over the distally based vessels. Subcutaneous tunnel is our preferred approach as it is simple and less time taking. Submuscular tunnel may be preferred to obtain more pedicle length in case of proximal abdominal wall defect reconstruction. The flap was allowed to perfuse 10 minutes before starting the inset. Flap thinning was done only at the flap margin to facilitate easy inset.
When the flap was based on the distal circulation, it was designed such that the pivot point of the flap was at least $5 \mathrm{~cm}$ above the knee joint. Flap harvest technique was similar as described above. Just before the division of the descending branch of LCFA proximally, it was temporarily occluded with microvascular clamp to confirm the perfusion from the distal circulation. Once the perfusion was satisfactory, the descending branch of LCFA was divided proximally and the flap was mobilised towards the knee. The proximal end of the LCFA vessels could be used to supercharge to great saphenous vein to prevent venous congestion.

\section{Case Series}

\section{Patient 1}

A 50-year-old male was diagnosed to have exstrophy of the bladder with fungating adenocarcinoma and epispadiasis (Figure 1) with bilateral inguinal lymph node metastasis. He underwent radical cystectomy and wide excision of the full thickness anterior abdominal wall, urinary diversion and bilateral inguinal lymphadenectomy. The resultant defect after partial primary closure of the abdominal wall superiorly was $17 \mathrm{~cm} \times 14$ $\mathrm{cm}$ (length $\mathrm{x}$ breadth) exposing the small bowel. Right thigh ALT flap was planned and harvested based on proximal circulation. The pedicle was tunnelled in the subcutaneous plane, the fascia lata was used to cover the fascial defect of the anterior abdominal wall, and skin suturing was done. Wound healed well and the patient recovery was uneventful.

\section{Patient 3}

A 38-year-old lady underwent excision of an unstable scar at the right iliac fossa, which was a sequela to the port site cancer, following treatment of urinary bladder cancer (Figure 2). After excision of the previous scar by the ablative surgeon, the defect was full thickness abdominal wall exposing the small bowel. Right thigh ALT flap was planned and the descending branch of LCFA was the pivot point. Fascial lata was used to reinforce the 

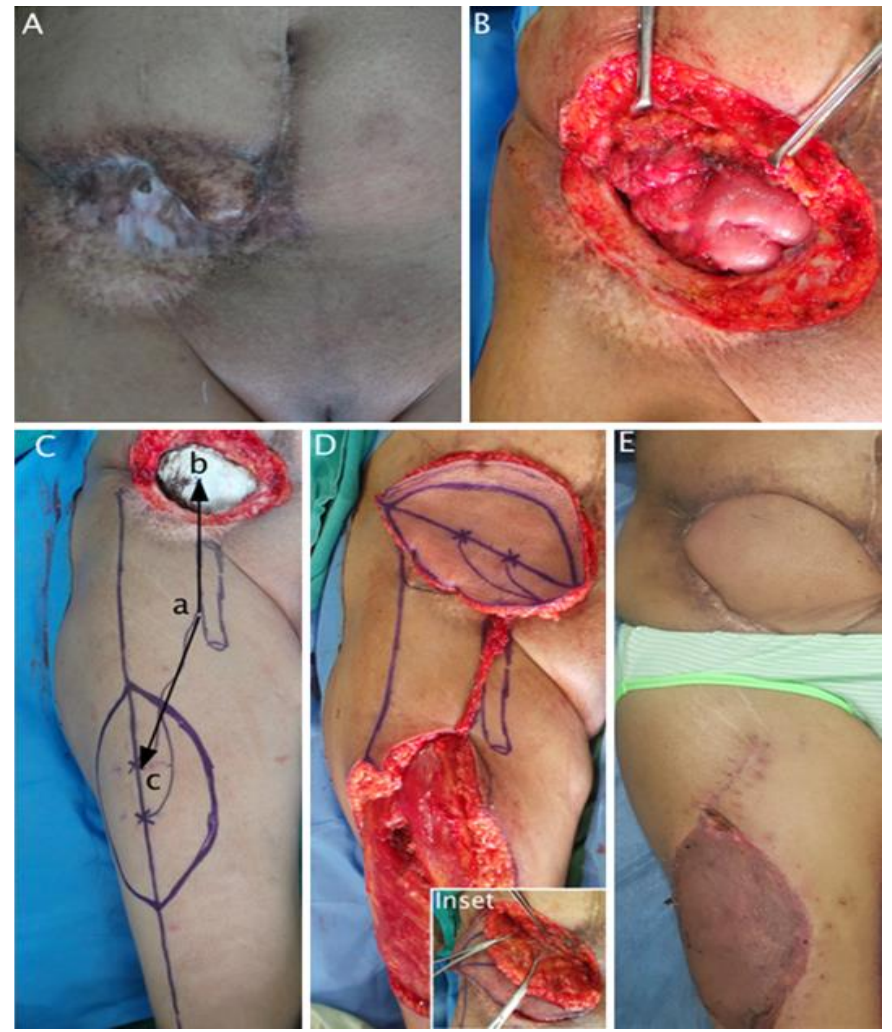

Figure 2. Image showing (A) unstable wound with underlying incisional hernia over the right iliac fossa region extending to the groin. (B) Composite abdominal wall defect extending to the right groin, following excision of the unstable wound. (C) Planning of proximally based pedicled ALT flap. Usually the pivot point ' $a$ ' is the origin of the descending branch of LCFA, ' $b$ ' is the mid-point at the defect, and ' $c$ ' is the location of the perforator to the ALT flap. Under ideal circumstances, $a b=b c$ provides tensionless flap inset. (D) Intraoperative image showing the reach of ALT flap to the recipient site. Image in the inset shows repair of the abdominal wall fascial defect with the fascia lata. (E) Healthy flap and donor site.

fascia of the abdominal wall and the inguinal ligament with prolene sutures. Pedicle was tunnelled under subcutaneous tissue to reach the defect. The donor site was covered with skin grafting. Postoperatively, the patient had purulent collection beneath the flap, and it was drained. Wound healed well; there was no hernia or bulge at the recipient site.

\section{Patient 4}

A 50-year-old female was referred to us for management of complicated soft tissue defect over the left trochanteric extending to left gluteal region. Earlier, she had sustained a road traffic accident and had fracture neck of femur and extensive Morel-Lavallee lesion over the left trochanteric and gluteal region, for which she underwent multiple sessions of debridement by general surgery service.

On examination, $20 \mathrm{~cm} \times 18 \mathrm{~cm}$ (length $\mathrm{x}$ breadth) wound was noted over the left trochanteric region, extending to the left gluteal region with undermining of the adjacent skin margins (Figure 3). She was also diagnosed to have left pubic rami fracture and fracture neck of femur.

The ALT thigh perforators were marked in the standard fashion and the LCFA was explored through the medial approach. Once the musculocutaneous perforator of descending branch of LCFA entry in to vastus lateralis (A) was identified, the distance between the perforator and the farthest wound margin (B) was measured $(25 \mathrm{~cm})$. The distal extent of the flap (C) was marked at $25 \mathrm{~cm}$ from the perforator and the flap was harvested in a musculocutaneous fashion to obliterate the cavity of the primary defect. Using the musculocutaneous perforator of descending branch of LCFA as the pivot point, the flap was rotated 150 degrees and

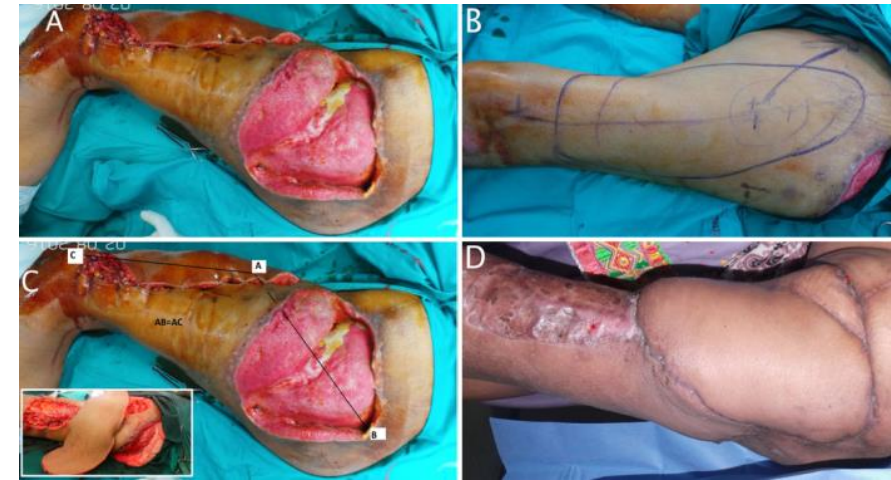

Figure 3. Image showing (A) $20 \mathrm{~cm} \times 18 \mathrm{~cm}$ (length $\mathrm{x}$ breadth) wound was noted over the left trochanteric region extending to the left gluteal region with undermining of the adjacent skin margins. (B) Marking of ipsilateral ALT flap. (C) Design of flap ' $A$ 'perforator of descending branch of LCFA entry in to vastus lateralis, ' $\mathrm{B}$ '- farthest point at the wound margin, ' $C$ '- distal point of the flap. The flap was designed such that $C A=A B$. Image in the inset showing islanded ALT flap, which is being propelled into the defect. (D) Healthy flap and well healed donor site skin grafting.
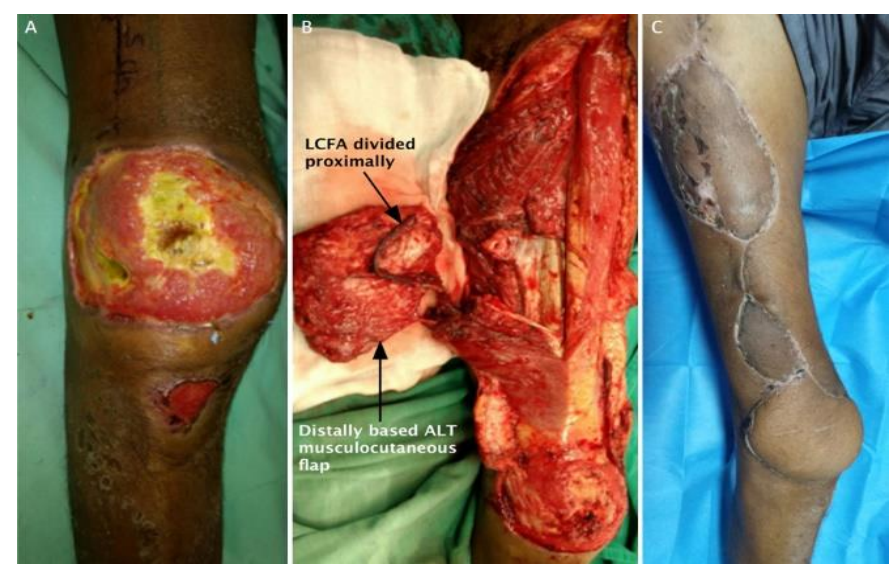

Figure 4. Image showing (A) right knee joint anterior surface with slough and exposed joint surface. (B) Distally based ALT flap detached from its proximal circulation. (C) Well settled flap and healed donor site.

inset was made after dividing the bridging segment between the primary defect and the flap. The secondary defect was covered with split thickness skin graft. Wounds were healed well and the flap was healthy at onemonth follow-up.

\section{Patient 6}

A 48-year-old male patient with peripheral vascular disease underwent aortofemoral bypass for femoral artery thrombosis. He developed soft tissue infection in the anterior aspect of the knee, which was debrided leading to exposure of the knee joint (Figure 4). Distally based ALT was planned. CT angiogram was done to confirm the presence of the ALT pedicle and the lateral superior genicular artery. ALT flap was designed islanded and the proximal descending branch of LCFA was clamped to check the perfusion of the flap from the distal circulation. Flap was rotated downwards to inset into the defect. The pivot point was placed $5 \mathrm{~cm}$ above the femoral condyles. The residual raw area was covered with skin grafting. Wounds healed uneventfully.

\section{Results}

The mean dimension of the defects included in our study was $12.5 \mathrm{~cm} \times$ $11.7 \mathrm{~cm}$ (range, 8 to $20 \times 5$ to $18 \mathrm{~cm}$ ). The rotations arcs are diagrammatically represented (Figure 4). All flaps were dissected sub-fascially. Abdominal fascia was restored in three patients. Early follow-up showed no 
significant hernia. All the perforators were musculocutaneous and all the patients required skin grafting at the ALT flap donor site. The mean followup duration was 5 months (range, 1 to 11 months)

Complications included one complete flap loss in patient 9 due to possible injury of lateral superior genicular vessels during the process of transarticular amputation. A CT angiogram to confirm the patency of line skeleton graph analysis (LSGA) could have been done to avoid the flap necrosis. This patient underwent skin grafting after multiple sessions of negative pressure wound therapy (NPWT) dressings. Two patients had partial flap loss. Patient 2 required debridement of the gangrenous skin component of the flap. The subcutaneous tissue and the fascia were intact. After few sessions of NPWT, the residual ulcer was covered with skin grafting. Patient 8 had tip gangrene at the knee joint, which was managed nonoperatively. Patient 3 had an abscess formation beneath the fascia of the flap, probably due to infected hematoma.

\section{Discussion}

Pedicled ALT flap is a versatile option for reconstruction of soft tissue defects within its reach. Many authors focussed the utility of pedicled ALT for various soft tissue defects pertaining to specific anatomical regions [47]. To the best of our knowledge, very few articles described the diverse anatomical locations where pedicled ALT flap was used, including the abdomen, groin, trochanteric region, and the knee [8].

The rotation arcs of the ALT flap in our series include the umbilicus superiorly, ipsilateral posterior superior iliac spine (PSIS) laterally, and tibial tuberosity below. Pedicled ALT flap can be utilized to cover the hand soft tissue defects in specific clinical scenarios. Lannol et al. described the largest series of the proximally pedicled ALT for abdominal and pelvic reconstruction. They described the farthest reach of the ALT flap i.e., lower costal margin, PSIS, anterior margin of anus, and contralateral iliac fossa. They also stressed upon the multiple factors like BMI, tunnel location, and flap design, which influenced the reach of the flap [9].

Wang et al. described pedicled ALT flap, which provided a reliable cover to the trochanteric region in the management of recurrent trochanteric pressure sores [10]. When the ALT flap was tunnelled through the muscular compartments of thigh, it could be utilised to resurface the ischial pressure ulcers [11]. Lee GK et al. emphasized the pedicled ALT as the preferred method for reconstruction of total penile defects. Pedicled ALT flap had several advantages over the free radial forearm flap, lack of need of microvascular surgery, better colour match, bulky flap, and less donor site morbidity [12]. Wong $S$ et al. had concluded that pedicled ALT with vastus lateralis muscle flap was a good alternative to vertical rectus abdominis myocutaneous flap for large defects associated with pelvic exenteration. The dead space could be obliterated by the bulk of the flap. The vagina and the perineum could be reconstructed sparing the integrity of the abdominal wall. Zelken JA et al. [13]. utilised pedicled ALT flap along with groin flap to reconstruct the mutilated hand injuries. ALT flap provided large amount of soft tissue cover along with the groin flap so that the circumferential degloving injuries could be reconstructed in a staged manner [14].

When based on the distally based circulation, ALT flap is a versatile option to cover the soft tissue defects around the knee joint and upper leg [15]. The fascia lata can be used to reconstruct the patellar ligament. Venous congestion is commonly expected when the flap is based on the distally based circulation, since the venous return needs to overcome the valves. The size of the flap can be increased and the venous congestion can be prevented by supercharging the flap vein to great saphenous vein [16]. We haven't faced the complication of venous congestion, possibly due to the small size of the flap we had used in our series. It has also been utilised to cover the defects involving the below knee stumps [17].
Split ALT flap was described to cover irregular wounds and to minimize wastage and trimming of flap [18]. When the ALT flap failed or the perforators could not be found, some back up procedures like tensor fascia latae flap or anteromedial thigh flap could be utilised [19]. Bulkiness of the flap was not a problem in our series. ALT flap was thin and pliable in most of the non-obese patients we encountered. All patients were satisfied with the contour of the flap postoperatively.

\section{Conclusion}

Pedicled anterolateral thigh flap is a versatile option for reconstruction of complex soft tissue defects in varied anatomical regions. Its wide arc of rotation and less donor site morbidity are its added advantages.

\section{Keywords}

Pedicled anterolateral thigh flap; versatility; islanded anterolateral thigh flap.

\section{Abbreviations}

ALT, anterolateral thigh flap; LCFA, lateral circumflex femoral artery; LSGA, line skeleton graph analysis; NPWT, negative pressure wound therapy; PSIS, posterior superior iliac spine.

\section{Article Information}

Conflict of Interest Disclosures: None

\section{Funding: None}

\section{References}

1. Song YG, Chen GZ, Song YL. The free thigh flap: A new free flap concept based on the septocutaneous artery. British Journal of Plastic Surgery 1984; 37: 149-159.

2. Wei FC, Jain V, Celik N, Chen HC, Chuang DC, Lin CH. Have we found an ideal soft-tissue flap? An experience with 672 anterolateral thigh flaps. Plastic and Reconstructive Surgery 2002; 109: 2219-2226; discussion 2227-2230.

3. Gravvanis Al, Tsoutsos DA, Karakitsos D, et al. Application of the pedicled anterolateral thigh flap to defects from the pelvis to the knee. Microsurgery 2006; 26: 432-438.

4. Friji MT, Suri MP, Shankhdhar VK, Ahmad QG, Yadav PS. Pedicled anterolateral thigh flap: a versatile flap for difficult regional soft tissue reconstruction. Annals of Plastic Surgery 2010; 64: 458-461.

5. Bharath SP, Madhusudan G, Manjunath S. Pedicled anterolateral thigh flap for contralateral groin composite defect. Indian Journal of Plastic Surgery 2010; 43: 103-105.

6. Gentileschi S, Servillo M, Garganese G, Simona F, Scambia G, Salgarello M. Versatility of pedicled anterolateral thigh flap in gynecologic reconstruction after vulvar cancer extirpative surgery. Microsurgery 2017; 37: 516-524.

7. Zelken JA, AlDeek NF, Hsu CC, Chang NJ, Lin CH, Lin CH. Algorithmic approach to lower abdominal, perineal, and groin reconstruction using anterolateral thigh flaps. Microsurgery 2016; 36: 104-114.

8. Lin $\mathrm{CT}$, Wang $\mathrm{CH}$, Ou KW, et al. Clinical applications of the pedicled anterolateral thigh flap in reconstruction. ANZ Journal of Surgery. 2017; 87: 499-504.

9. Lannon DA, Ross GL, Addison PD, Novak CB, Lipa JE, Neligan PC. Versatility of the proximally pedicled anterolateral thigh flap and its use in complex abdominal and pelvic reconstruction. Plastic and Reconstructive Surgery 2011; 127:677-688. 
10. Wang $\mathrm{CH}$, Chen SY, Fu JP, et al. Reconstruction of trochanteric pressure sores with pedicled anterolateral thigh myocutaneous flaps. Journal of Plastic, Reconstructive \& Aesthetic Surgery 2011; 64: 671676.

11. Lee JT, Cheng LF, Lin CM, Wang $\mathrm{CH}$, Huang CC, Chien SH. A new technique of transferring island pedicled anterolateral thigh and vastus lateralis myocutaneous flaps for reconstruction of recurrent ischial pressure sores. Journal of Plastic, Reconstructive \& Aesthetic Surgery 2007; 60: 1060-1066.

12. Lee GK, Lim AF, Bird ET. A novel single-flap technique for total penile reconstruction: the pedicled anterolateral thigh flap. Plastic and Reconstructive Surgeryz 2009; 124: 163-166.

13. Wong S, Garvey P, Skibber J, Yu P. Reconstruction of pelvic exenteration defects with anterolateral thigh-vastus lateralis muscle flaps. Plastic and Reconstructive Surgery 2009; 124: 1177-1185.

14. Zelken JA, Chang NJ, Wei FC, Lin CH. The combined ALT-groin flap for the mutilated and degloved hand. Injury 2015; 46: 1591-1596.

15. Pan SC, Yu JC, Shieh SJ, Lee JW, Huang BM, Chiu HY. Distally based anterolateral thigh flap: an anatomic and clinical study. Plastic and Reconstructive Surgery 2004; 114: 1768-1775.

16. Lin $\mathrm{CH}$, Zelken J, Hsu CC, Lin $\mathrm{CH}$, Wei FC. The distally based, venous supercharged anterolateral thigh flap. Microsurgery 2016; 36: 20-28.

17. Sadigh PL, Wu C-J, Shih H-S, Jeng S-F. Reverse Anterolateral Thigh Flap to Revise a Below-knee Amputation Stump at the Mid-tibial Level. Plastic and Reconstructive Surgery Global Open 2013; 1: e88.

18. Chang NJ, Waughlock N, Kao D, Lin CH, Lin CH, Hsu CC. Efficient design of split anterolateral thigh flap in extremity reconstruction. Plastic and Reconstructive Surgery 2011 Dec; 128: 1242-1249.

19. Hsieh $\mathrm{CH}$, Yang JC, Chen CC, Kuo YR, Jeng SF. Alternative reconstructive choices for anterolateral thigh flap dissection in cases in which no sizable skin perforator is available. Head Neck 2009; 31: 571-575. 